\title{
Surgical Margins during Hepatic Surgery for Colorectal Liver Metastases: Complete Resection not Millimeters Defines Outcome
}

\author{
Timothy M. Pawlik, MD MPH, ${ }^{1}$ and Jean-Nicolas Vauthey, $\mathrm{MD}^{2}$ \\ ${ }^{1}$ Department of Surgery, Johns Hopkins Hospital, Maryland, USA \\ ${ }^{2}$ Department of Surgical Oncology, Box 444, The University of Texas M.D. Anderson Cancer Center, 1515 Holcombe Boulevard, \\ Houston, Texas 77030, USA
}

Several recent studies have indicated that a margin less than $1 \mathrm{~cm}$ is not a contraindication to resection of colorectal liver metastases (CLM). ${ }^{1-3}$ In the one study ${ }^{2}$ that appropriately excluded from analyses those patients with positive margins, only number of metastases and extrahepatic disease - not margin width less than $1 \mathrm{~cm}$ - predicted outcome. In our own series of 557 patients undergoing resection of CLM, patients with a less-than-5-mm negative margin who underwent resection infrequently experienced recurrence locally $(5 \%)$. Of note, the incidence of local recurrence was similarly uncommon $(11 \%)$ in patients resected who had positive margins. Important, however, was the finding that only $17 \%$ of patients with a positive margin were alive after 5 years. ${ }^{1}$ In contrast, those patients with a negative margin had a 5-year survival of $64 \%$, regardless of the width of the surgical margin. In multivariate analysis, tumor number greater than 3 and tumor size equal to or more than $5 \mathrm{~cm}$, but not positive margins, were the main predictors of adverse outcome. In aggregate, these data strongly suggest that aggressive biological factors, and not necessarily margin status itself, dictate outcome following hepatic resection of CLMs. While adequate surgical margins are clearly important, the actual width may not be as

Received October 23, 2007; accepted October 24, 2007; published online: December 29, 2007.

Address correspondence and reprint requests to: Jean-Nicolas Vauthey, MD; E-mail: jvauthey@mdanderson.org

Published by Springer Science+Business Media, LLC $\odot 2007$ The Society of Surgical Oncology, Inc. critical in resecting CLMs because these lesions are histopathologically well circumscribed, ${ }^{4}$ only $16 \%$ have satellitosis, ${ }^{5}$ Glisson sheath extension is uncommon (14.5\%) and limited (5 $\mathrm{mm}$ or less), ${ }^{6}$ and micrometastases are rare $(2 \%){ }^{6}$ As such, the currently available data support the concept of limited complete resection utilizing a planned approach that encompasses all tumor-bearing liver parenchyma.

In this current issue of Annals of Surgical Oncology, Elias et al. address the issue of surgical margin as it relates to preoperative cross-sectional imaging. Specifically, Elias et al. have compared the preoperative margins as defined by computed tomography and the final margins as defined by pathological evaluation of the resection specimen. The authors note that approximately 5-8 $\mathrm{mm}$ of tumor-free hepatic parenchyma will "disappear" during hepatectomy. Given this, the authors recommend a planned preoperative surgical margin of 7-10 mm using preoperative computed tomography. However, many factors may have contributed to the difference between the radiological measurement of margins using preoperative computed tomography and the surgical margin as measured based on the pathology specimen.

Evaluation of preoperative margins is directly related to image quality. Thin cuts $(2.5 \mathrm{~mm})$, high quality bolus of intravenous contrast and appropriate scanning delays (ideally triple-phase, liver imaging technique) are needed to assure accurate definition of tumor contours and enhancement of the hepatic veins. It is unclear whether Elias et al. used a uniform 
imaging technique, which would have provided the optimal evaluation of the tumor contours and the tumor-major hepatic veins relationship. The authors also used the "crushing technique" of parenchymal transection, which does not necessarily minimize tissue loss and tumor exposure. Some "disappearance" of the tumor-free margin may, therefore, be attributable to the chosen technique of hepatic transection. In our series of 557 liver resections using mostly the ultrasonic dissector for parenchymal transection, ${ }^{1}$ we had a margin-positive rate of only $8 \%$, which compares favorably to other series. ${ }^{3}$ As such, whether the findings of the report by Elias et al. are broadly applicable to patients undergoing hepatic resection using techniques other than the "crushing technique" remains undefined. Elias et al. also note that resection occurred "within $3 \mathrm{~mm}$ " of the middle hepatic vein; however, the middle hepatic vein along Cantlie's line was not universally and strictly exposed. Frequently, main tributaries to the middle hepatic vein or the cephalad extent of the dissection near the hepatocaval junction can cause the surgeon to course away from the middle hepatic vein toward the right or left portal fissure planes during the dissection. Finally, while the authors mention the use of intraoperative ultrasound (IOUS), they do not report the distance between the tumor and the middle hepatic vein as measured by IOUS. This is important, as IOUS arguably is the modality that most accurately defines the tumorvessel relationship. Using ultrasonography-guided liver resection, Torzilli et al. $^{7}$ recently advocated resection of the hepatic vein only when tumor was inseparable from the vessel wall. They reported a $0 \%$ local recurrence after a median follow-up of 18 months.

What is the most efficient surgical technique and what are the pitfalls to avoid incomplete resection of hepatic CLMs? The combined use of preoperative and intraoperative imaging is critical to define and clarify the tumor-vessel relationship. If the portal phase of the preoperative computed tomography does not define the relationship between the tumor and the vein (which can occur in cases of severe steatosis and hemodynamic variations affecting contrast enhancement of the hepatic veins), a magnetic resonance image should be obtained. If the hepatic vein is involved (e.g., tumor is inseparable from the hepatic vein) a planned resection including the middle hepatic vein should strongly be considered. This may involve a central resection, ${ }^{8}$ an extended resection after portal vein embolization ${ }^{9}$ or a segmental resection of the hepatic vein if reversed portal flow is demonstrated intraoperatively. ${ }^{10}$
If on preoperative imaging the tumor is close to the middle hepatic vein, IOUS should ultimately define the surgical approach. In this context, we would not consider radiofrequency-assisted metastasectomy, as advocated by Elias et al. Radiofrequency ablation can be associated with distortion of the hepatic parenchyma along the course of the major vessel, thereby making dissection along this plane more difficult. We therefore prefer the technique that was recently described as the "two-surgeon technique", which allows for dissection along the hepatic veins (with or without extrahepatic control of the vein at the hepatocaval junction). ${ }^{11}$ This technique of parenchymal transection combines the saline-linked cautery and the ultrasonic dissector, and is associated with reduced blood loss as well as shorter parenchymal transection times. In addition, the "two-surgeon technique" optimizes the surgical exposure and facilitates dissection along the hepatic veins up to the hepatocaval junction.

Elias et al. should be congratulated for emphasizing the need for a systematic planned approach to achieve a margin-negative (curative) resection. The surgical strategies outlined above should contribute to achieving this goal.

\section{REFERENCES}

1. Pawlik TM, Scoggins CR, Zorzi D, et al. Effect of surgical margin status on survival and site of recurrence after hepatic resection for colorectal metastases. Ann Surg 2005; 241:71524.

2. Figueras J, Burdio F, Ramos E, et al. Effect of subcentimeter nonpositive resection margin on hepatic recurrence in patients undergoing hepatectomy for colorectal liver metastases. Evidences from 663 liver resections. Ann Oncol 2007; 18:1190-5.

3. Are C, Gonen M, Zazzali K, et al. The impact of margins on outcome after hepatic resection for colorectal metastasis. Ann Surg 2007; 246:295-300.

4. Yamamoto J, Sugihara K, Kosuge T, et al. Pathologic support for limited hepatectomy in the treatment of liver metastases from colorectal cancer. Ann Surg 1995; 221:74-8.

5. Scheele J, Stang R, Altendorf-Hofmann A, et al. Resection of colorectal liver metastases. World J Surg 1995; 19:59-71.

6. Kokudo N, Miki Y, Sugai S, et al. Genetic and histological assessment of surgical margins in resected liver metastases from colorectal carcinoma: minimum surgical margins for successful resection. Arch Surg 2002; 137:833-40.

7. Torzilli G, Montorsi M, Donadon M, et al. "Radical but conservative" is the main goal for ultrasonography-guided liver resection: prospective validation of this approach. $J \mathrm{Am}$ Coll Surg 2005; 201:517-28.

8. Chouillard E, Cherqui D, Tayar C, et al. Anatomical bi- and trisegmentectomies as alternatives to extensive liver resections. Ann Surg 2003; 238:29-34.

9. Vauthey JN, Pawlik TM, Abdalla EK, et al. Is extended hepatectomy for hepatobiliary malignancy justified?. Ann Surg 2004; 239:722-32. 
10. Torzilli G, Montorsi M, Fabbro DD, Palmisano A, Donandon M, Makuuchi M.. Ultrasonographically guided surgical approach to liver tumors involving the hepatic veins close to the caval confluence. Br J Surg 2006; 93:1238-46.
11. Aloia TA, Zorzi D, Abdalla EK, et al. Two-surgeon technique for hepatic parenchymal transection of the noncirrhotic liver using saline-linked cautery and ultrasonic dissection. Ann Surg $2005 ; 242: 172-7$. 